\title{
Relaxing the Cosmological Constraints on Unparticle Dark Component
}

\author{
Hao Wei* \\ Department of Physics, Beijing Institute of Technology, Beijing 100081, China
}

\begin{abstract}
Unparticle physics has been an active field since the seminal work of Georgi. Recently, many constraints on unparticles from various observations have been considered in the literature. In particular, the cosmological constraints on the unparticle dark component put it in a serious situation. In this work, we try to find a way out of this serious situation, by including the possible interaction between dark energy and the unparticle dark component.
\end{abstract}

PACS numbers: 98.80.Es, 14.80.-j, 95.36.+x, 98.80.-k 


\section{INTRODUCTION}

Recently, the so-called unparticle physics has been an active field since the seminal work of Georgi 1, 2]. It was based on the hypothesis that there could be an exact scale invariant hidden sector resisted at a high energy scale. A prototype model of such a sector is given by the Banks-Zaks theory which flows to an infrared fixed point at a lower energy scale $\Lambda_{\mathrm{u}}$ through dimensional transmutation [3]. Recently, there has been a flood of papers on the unparticle phenomenology. We refer to e.g. 3, 4] for some brief reviews. In fact, soon after the seminal work of Georgi 1, 2], some authors considered the constraints on unparticles from various observations, such as the collider experiments [5], the new long range force experiments [6, [7], the solar and reactor neutrinos data [8], as well as the observations from astrophysics and cosmology [7, 9, 10, 11, 23]. These observations put fairly stringent constraints on the unparticle.

The unparticle does not have a definite mass and instead has a continuous spectral density as a consequence of scale invariance [1, 2] (see also e.g. [11, 12, 24]),

$$
\rho\left(P^{2}\right)=A_{d_{\mathrm{u}}} \theta\left(P^{0}\right) \theta\left(P^{2}\right)\left(P^{2}\right)^{d_{\mathrm{u}}-2},
$$

where $P$ is the 4 -momentum; $A_{d_{\mathrm{u}}}$ is the normalization factor; $d_{\mathrm{u}}$ is the scaling dimension. When $d_{\mathrm{u}}=1$, Eq. (11) reduces to the familiar one of a massless particle. The scaling dimension $d_{\mathrm{u}}$ is constrained by the unitarity of the conformal algebra and the requirement to avoid the singular behavior [1, 4] . The theoretical bounds are $1 \leq d_{\mathrm{u}} \leq 2$ (for bosonic unparticles) or $3 / 2 \leq d_{\mathrm{u}} \leq 5 / 2$ (for fermionic unparticles) [4]. Typically, values $1 \leq d_{\mathrm{u}} \leq 2$ have been extensively considered in the literature.

The pressure and energy density of thermal unparticles have been derived in [12]. They are

$$
\begin{aligned}
& p_{\mathrm{u}}=g_{s} T^{4}\left(\frac{T}{\Lambda_{\mathrm{u}}}\right)^{2\left(d_{\mathrm{u}}-1\right)} \frac{\mathcal{C}\left(d_{\mathrm{u}}\right)}{4 \pi^{2}}, \\
& \rho_{\mathrm{u}}=\left(2 d_{\mathrm{u}}+1\right) g_{s} T^{4}\left(\frac{T}{\Lambda_{\mathrm{u}}}\right)^{2\left(d_{\mathrm{u}}-1\right)} \frac{\mathcal{C}\left(d_{\mathrm{u}}\right)}{4 \pi^{2}},
\end{aligned}
$$

where $\mathcal{C}\left(d_{\mathrm{u}}\right)=B\left(3 / 2, d_{\mathrm{u}}\right) \Gamma\left(2 d_{\mathrm{u}}+2\right) \zeta\left(2 d_{\mathrm{u}}+2\right)$, while $B, \Gamma, \zeta$ are the Beta, Gamma and Zeta functions, respectively. These are the results for the bosonic unparticles. The pressure and energy density of fermionic unparticles can be obtained by replacing $\mathcal{C}\left(d_{\mathrm{u}}\right)$ by $\left[1-2^{-\left(2 d_{\mathrm{u}}+1\right)}\right] \mathcal{C}\left(d_{\mathrm{u}}\right)$. Therefore, the equation-of-state parameter (EoS) of both the bosonic and fermionic unparticles is given by [12]

$$
w_{\mathrm{u}} \equiv \frac{p_{\mathrm{u}}}{\rho_{\mathrm{u}}}=\frac{1}{2 d_{\mathrm{u}}+1} .
$$

When $d_{\mathrm{u}}=1$, we have $w_{\mathrm{u}}=1 / 3$, which is the same as radiation. When $d_{\mathrm{u}} \rightarrow \infty$, we find $w_{\mathrm{u}} \rightarrow 0$, which approaches the EoS of pressureless matter. In the intermediate case, the EoS of unparticles is different from the one of radiation or cold dark matter, and generically lies in between. Since the unparticle interacts weakly with standard model particles, it is "dark" in this sense. Some authors regard the unparticle as a candidate of dark matter [13] (see also [11, 12]). Noting that the EoS of unparticles $\left(w_{\mathrm{u}}>0\right)$ is different from the one of cold dark matter $\left(w_{\mathrm{m}}=0\right)$, we instead call it "dark component" to avoid confusion.

In [11], the cosmological constraints on the unparticle dark component have been considered, by using the type Ia supernovae (SNIa), the shift parameter of the cosmic microwave background (CMB), and the baryon acoustic oscillation (BAO). The authors of [11] found that $d_{\mathrm{u}}>60$ at $95 \%$ confidence level (C.L.) for the $\Lambda \mathrm{UDM}$ model in which the unparticle is the sole dark matter. As mentioned above, however, the theoretical bounds on unparticles are $1 \leq d_{\mathrm{u}} \leq 2$ (for bosonic unparticles) or $3 / 2 \leq d_{\mathrm{u}} \leq 5 / 2$ (for fermionic unparticles) [4]. The situation is serious. Even for the $\Lambda \mathrm{UCDM}$ model in which the unparticle dark component co-exists with cold dark matter, they found that the unparticle dark component can at most make up a few percent of the total cosmic energy density if $d_{\mathrm{u}}<10$; so that it cannot be a major component 11. In fact, it is easy to understand these results. Since the unparticle dark component scales as $a^{-3\left(1+w_{\mathrm{u}}\right)}$ whereas cold dark matter scales as $a^{-3}$ (here $a$ is the scale factor of the universe), for $w_{\mathrm{u}}>0$, the energy density of the unparticle dark component decreases faster than the one of cold dark matter. So, it is not surprising that the energy density of the unparticle dark component cannot be comparable 
with cold dark matter at the present epoch. To make a considerable contribution to the total cosmic energy density, $d_{\mathrm{u}}$ should be very large to make $w_{\mathrm{u}} \simeq 0$ so that the unparticle dark component could mimic cold dark matter.

In this work, we try to find a way out of the serious situation mentioned above. Our physical motivation is very simple. If dark energy, the major component of the universe, can decay into unparticles, the energy density of the unparticle dark component should decrease more slowly. Since both dark energy and unparticle are unseen, the interaction between them is not prevented. If the dilution rates of unparticle dark component and cold dark matter are comparable, it is possible to have a considerable energy density of the unparticle dark component at the present epoch, without requiring $d_{\mathrm{u}}$ to be very large. In fact, this is similar to the key point of the interacting dark energy models which are extensively considered in the literature to alleviate the cosmological coincidence problem (see e.g. [14, 25] and references therein).

Here, we consider a flat universe which contains dark energy, the unparticle dark component and pressureless matter (including cold dark matter and baryons). We assume that dark energy and unparticle dark component exchange energy according to (see e.g. [14, 25] and references therein)

$$
\begin{aligned}
& \dot{\rho}_{\mathrm{de}}+3 H \rho_{\mathrm{de}}\left(1+w_{\mathrm{de}}\right)=-Q, \\
& \dot{\rho}_{\mathrm{u}}+3 H \rho_{\mathrm{u}}\left(1+w_{\mathrm{u}}\right)=Q
\end{aligned}
$$

whereas pressureless matter (including cold dark matter and baryons) evolves independently, i.e.,

$$
\dot{\rho}_{\mathrm{m}}+3 H \rho_{\mathrm{m}}=0 \text {. }
$$

So, the total energy conservation equation $\dot{\rho}_{\text {tot }}+3 H \rho_{\text {tot }}\left(1+w_{\text {tot }}\right)=0$ is preserved. $H \equiv \dot{a} / a$ is the Hubble parameter; $a=(1+z)^{-1}$ is the scale factor of the universe (we set $a_{0}=1$ ); the subscript " 0 " indicates the present value of the corresponding quantity; $z$ is the redshift; a dot denotes the derivative with respect to cosmic time $t$. The interaction forms extensively considered in the literature (see for instance [14, 25] and references therein) are $Q \propto H \rho_{\mathrm{m}}, H \rho_{\mathrm{de}}, H \rho_{\mathrm{tot}}, \kappa \rho_{\mathrm{m}} \dot{\phi}$ (where $\kappa^{2} \equiv 8 \pi G$ ), and so on. In this work, for simplicity, we consider the interaction term

$$
Q=3 \alpha H \rho_{\mathrm{u}}
$$

where $\alpha$ is a constant. So, Eq. (6) becomes $\dot{\rho}_{\mathrm{u}}+3 H \rho_{\mathrm{u}}\left(1+w_{\mathrm{u}}^{\mathrm{eff}}\right)=0$, where $w_{\mathrm{u}}^{\mathrm{eff}} \equiv w_{\mathrm{u}}-\alpha$. It is easy to find that

$$
\rho_{\mathrm{u}}=\rho_{\mathrm{u} 0} a^{-3\left(1+w_{\mathrm{u}}^{\mathrm{eff}}\right)} .
$$

On the other hand, from Eq. (7), we have $\rho_{\mathrm{m}}=\rho_{\mathrm{m} 0} a^{-3}$. For convenience, we introduce the fractional energy density $\Omega_{i} \equiv\left(8 \pi G \rho_{i}\right) /\left(3 H^{2}\right)$, where $i=$ de, $\mathrm{u}$ and $\mathrm{m}$.

In the following sections, similar to [11], we consider the cosmological constraints on the unparticle dark component which interacts with dark energy, by using the observations of SNIa, the shift parameter $R$ of CMB, and the distance parameter $A$ from BAO. In Sec. III, we present the cosmological data and the methodology used to constrain the models. In Sec. III and IV] we consider the observational constraints on the interacting $\Lambda \mathrm{UCDM}$ model and the interacting XUCDM model, respectively. As expected, we find that the cosmological constraints on the unparticle dark component can be significantly relaxed, thanks to the possible interaction between dark energy and unparticle dark component. The tension between theoretical bounds and cosmological constraints could be removed. Finally, some concluding remarks are given in Sec. V

\section{COSMOLOGICAL DATA}

In this work, we perform a $\chi^{2}$ analysis to obtain the constraints on the model parameters. The data points of the 307 Union SNIa compiled in [15] are given in terms of the distance modulus $\mu_{o b s}\left(z_{i}\right)$. On the other hand, the theoretical distance modulus is defined as

$$
\mu_{t h}\left(z_{i}\right) \equiv 5 \log _{10} D_{L}\left(z_{i}\right)+\mu_{0}
$$


where $\mu_{0} \equiv 42.38-5 \log _{10} h$ and $h$ is the Hubble constant $H_{0}$ in units of $100 \mathrm{~km} / \mathrm{s} / \mathrm{Mpc}$, whereas

$$
D_{L}(z)=(1+z) \int_{0}^{z} \frac{d \tilde{z}}{E(\tilde{z} ; \mathbf{p})},
$$

in which $E \equiv H / H_{0} ; \mathbf{p}$ denotes the model parameters. The $\chi^{2}$ from the 307 Union SNIa are given by

$$
\chi_{\mu}^{2}(\mathbf{p})=\sum_{i} \frac{\left[\mu_{o b s}\left(z_{i}\right)-\mu_{t h}\left(z_{i}\right)\right]^{2}}{\sigma^{2}\left(z_{i}\right)},
$$

where $\sigma$ is the corresponding $1 \sigma$ error. The parameter $\mu_{0}$ is a nuisance parameter but it is independent of the data points. One can perform an uniform marginalization over $\mu_{0}$. However, there is an alternative way. Following [16, 17], the minimization with respect to $\mu_{0}$ can be made by expanding the $\chi_{\mu}^{2}$ of Eq. (12) with respect to $\mu_{0}$ as

$$
\chi_{\mu}^{2}(\mathbf{p})=\tilde{A}-2 \mu_{0} \tilde{B}+\mu_{0}^{2} \tilde{C},
$$

where

$$
\begin{gathered}
\tilde{A}(\mathbf{p})=\sum_{i} \frac{\left[\mu_{o b s}\left(z_{i}\right)-\mu_{t h}\left(z_{i} ; \mu_{0}=0, \mathbf{p}\right)\right]^{2}}{\sigma_{\mu_{o b s}}^{2}\left(z_{i}\right)} \\
\tilde{B}(\mathbf{p})=\sum_{i} \frac{\mu_{o b s}\left(z_{i}\right)-\mu_{t h}\left(z_{i} ; \mu_{0}=0, \mathbf{p}\right)}{\sigma_{\mu_{o b s}}^{2}\left(z_{i}\right)}, \quad \tilde{C}=\sum_{i} \frac{1}{\sigma_{\mu_{o b s}}^{2}\left(z_{i}\right)} .
\end{gathered}
$$

Eq. (13) has a minimum for $\mu_{0}=\tilde{B} / \tilde{C}$ at

$$
\tilde{\chi}_{\mu}^{2}(\mathbf{p})=\tilde{A}(\mathbf{p})-\frac{\tilde{B}(\mathbf{p})^{2}}{\tilde{C}} .
$$

Since $\chi_{\mu, \min }^{2}=\tilde{\chi}_{\mu, \min }^{2}$ obviously, we can instead minimize $\tilde{\chi}_{\mu}^{2}$ which is independent of $\mu_{0}$. The shift parameter $R$ of $\mathrm{CMB}$ is defined by [18, 19]

$$
R \equiv \Omega_{\mathrm{m} 0}^{1 / 2} \int_{0}^{z_{*}} \frac{d \tilde{z}}{E(\tilde{z})},
$$

where the redshift of recombination $z_{*}=1090$ which has been updated in the Wilkinson Microwave Anisotropy Probe 5-year (WMAP5) data 20]. The shift parameter $R$ relates the angular diameter distance to the last scattering surface, the comoving size of the sound horizon at $z_{*}$ and the angular scale of the first acoustic peak in CMB power spectrum of temperature fluctuations [18, 19]. The value of $R$ has been updated to $1.710 \pm 0.019$ from the WMAP5 data [20]. On the other hand, the distance parameter $A$ of the measurement of the BAO peak in the distribution of SDSS luminous red galaxies [21] is given by

$$
A \equiv \Omega_{\mathrm{m} 0}^{1 / 2} E\left(z_{b}\right)^{-1 / 3}\left[\frac{1}{z_{b}} \int_{0}^{z_{b}} \frac{d \tilde{z}}{E(\tilde{z})}\right]^{2 / 3},
$$

where $z_{b}=0.35$. In 22], the value of $A$ has been determined to be $0.469\left(n_{s} / 0.98\right)^{-0.35} \pm 0.017$. Here the scalar spectral index $n_{s}$ is taken to be 0.960 , which has been updated from the WMAP5 data [20]. So, the total $\chi^{2}$ is given by

$$
\chi^{2}=\tilde{\chi}_{\mu}^{2}+\chi_{C M B}^{2}+\chi_{B A O}^{2},
$$

where $\tilde{\chi}_{\mu}^{2}$ is given in Eq. (14), $\chi_{C M B}^{2}=\left(R-R_{o b s}\right)^{2} / \sigma_{R}^{2}$ and $\chi_{B A O}^{2}=\left(A-A_{o b s}\right)^{2} / \sigma_{A}^{2}$. The best-fit model parameters are determined by minimizing the total $\chi^{2}$. As is well known, the $68 \%$ C.L. is determined by $\Delta \chi^{2} \equiv \chi^{2}-\chi_{\min }^{2} \leq 1.00,2.30,3.53,4.72$ and 5.89 for $n=1,2,3,4$ and 5, respectively, where $n$ is the number of free model parameters. Similarly, the $95 \%$ C.L. is determined by $\Delta \chi^{2} \equiv \chi^{2}-\chi_{\min }^{2} \leq 4.00$, $6.17,8.02,9.70$ and 11.3 for $n=1,2,3,4$ and 5 , respectively.

In the following sections, we consider the observational constraints on the interacting $\Lambda$ UCDM model and the interacting XUCDM model, respectively, by using the methodology presented here. 


\section{COSMOLOGICAL CONSTRAINTS ON THE INTERACTING $\Lambda$ UCDM MODEL}

In this section, we consider the interacting $\Lambda \mathrm{UCDM}$ model in which the role of dark energy is played by the decaying cosmological constant (vacuum energy), as in the well-known $\Lambda(t) \mathrm{CDM}$ model [26, 27, 28]. In this case, Eq. (5) becomes

$$
\dot{\rho}_{\Lambda}=-3 \alpha H \rho_{\mathrm{u}}=-3 \alpha \rho_{\mathrm{u} 0} H a^{-3\left(1+w_{\mathrm{u}}^{\text {eff }}\right)} .
$$

Noting that the case without interaction $(\alpha=0)$ was considered in [11], there are two other different cases with $w_{\mathrm{u}}^{\mathrm{eff}} \neq-1$ and $w_{\mathrm{u}}^{\mathrm{eff}}=-1$ for this differential equation. We will study them one by one.
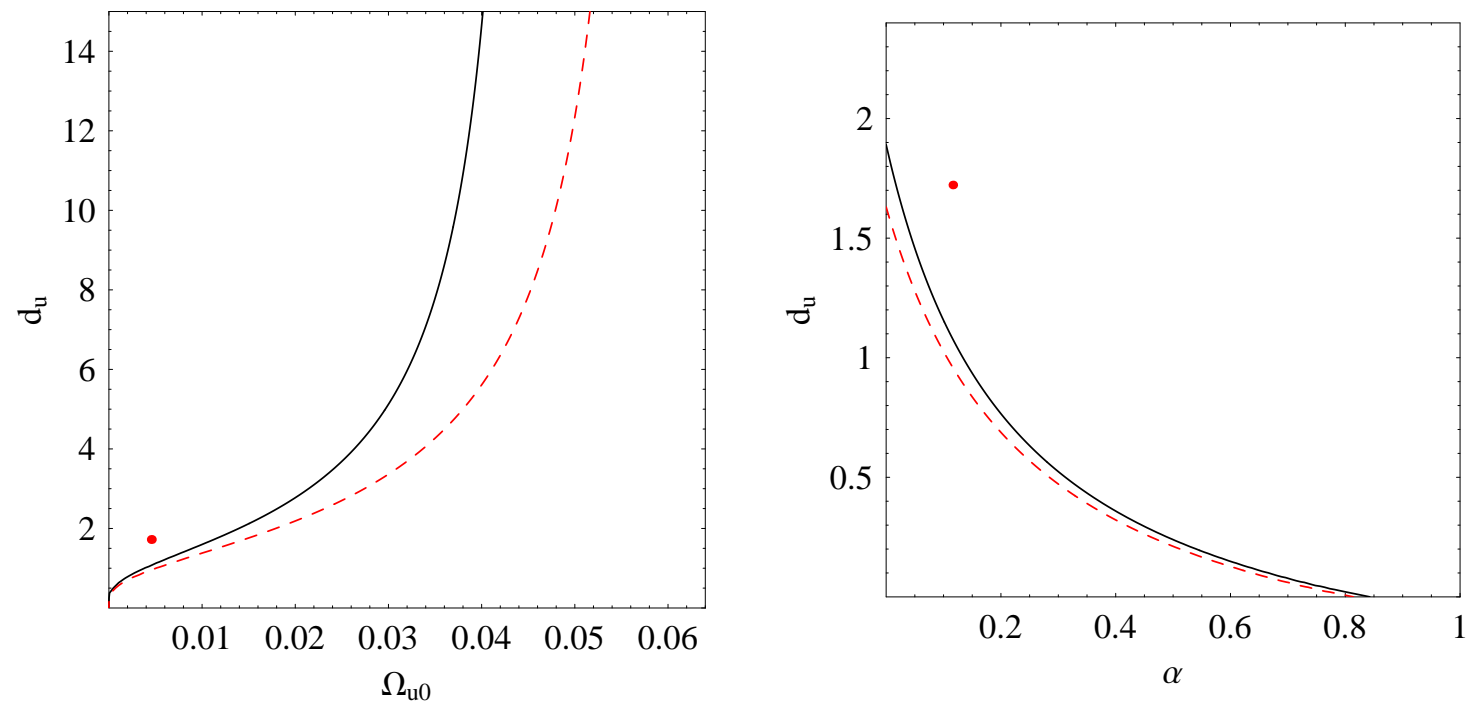

FIG. 1: The $68 \%$ C.L. (black solid lines) and $95 \%$ C.L. (red dashed lines) contours in the $\Omega_{\mathrm{u} 0}-d_{\mathrm{u}}$ plane and the $\alpha-d_{\mathrm{u}}$ plane for the interacting $\Lambda \mathrm{UCDM}$ model with $w_{\mathrm{u}}^{\mathrm{eff}} \neq-1$. The best fit is indicated by the red solid point.

\section{A. The interacting $\Lambda \mathrm{UCDM}$ model with $w_{\mathrm{u}}^{\mathrm{eff}} \neq-1$}

If $w_{\mathrm{u}}^{\mathrm{eff}} \neq-1$, the solution for Eq. (18) is given by

$$
\rho_{\Lambda}=\frac{\alpha \rho_{\mathrm{u} 0}}{1+w_{\mathrm{u}}^{\text {eff }}} a^{-3\left(1+w_{\mathrm{u}}^{\mathrm{eff}}\right)}+\text { const. },
$$

where const. is an integration constant. Obviously, this solution diverges for $w_{\mathrm{u}}^{\mathrm{eff}}=-1$ which will be considered in the next subsection. Inserting Eq. (19) into the Friedmann equation $H^{2}=\kappa^{2}\left(\rho_{\Lambda}+\rho_{\mathrm{u}}+\rho_{\mathrm{m}}\right) / 3$ and requiring $E(z=0)=1$, one can determine the integration constant. Finally, we find that

$$
\left.E^{2}=\Omega_{\mathrm{m} 0}(1+z)^{3}+\left(1+\frac{\alpha}{1+w_{\mathrm{u}}^{\text {eff }}}\right) \Omega_{\mathrm{u} 0}(1+z)^{3\left(1+w_{\mathrm{u}}^{\text {eff }}\right.}\right)+\left[1-\Omega_{\mathrm{m} 0}-\left(1+\frac{\alpha}{1+w_{\mathrm{u}}^{\text {eff }}}\right) \Omega_{\mathrm{u} 0}\right] .
$$

There are 4 free model parameters, namely, $\Omega_{\mathrm{m} 0}, \Omega_{\mathrm{u} 0}, d_{\mathrm{u}}$ and $\alpha$. By minimizing the corresponding total $\chi^{2}$ in Eq. (17), we find the best-fit parameters $\Omega_{\mathrm{m} 0}=0.280, \Omega_{\mathrm{u} 0}=0.005, d_{\mathrm{u}}=1.722$ and $\alpha=0.116$, while $\chi_{\min }^{2}=311.924$. In Fig. 1, we present the corresponding $68 \%$ and $95 \%$ C.L. contours in the $\Omega_{\mathrm{u} 0}-d_{\mathrm{u}}$ plane and the $\alpha-d_{\mathrm{u}}$ plane for the interacting $\Lambda \mathrm{UCDM}$ model with $w_{\mathrm{u}}^{\mathrm{eff}} \neq-1$, while the other parameters are taken to be the corresponding best-fit values. Obviously, from the left panel of Fig. [1 we see that the unparticle dark component can at most make up a few percent of the total cosmic energy density, so that it cannot be a major component in this case. 


\section{B. The interacting $\Lambda \mathrm{UCDM}$ model with $w_{\mathrm{u}}^{\mathrm{eff}}=-1$}

As mentioned above, the solution Eq. (19) diverges for $w_{\mathrm{u}}^{\text {eff }}=-1$. Therefore, one is required to consider this case separately. If $w_{\mathrm{u}}^{\text {eff }}=-1$, we find that

$$
\alpha=1+w_{\mathrm{u}}=1+\frac{1}{2 d_{\mathrm{u}}+1} .
$$

So, $\alpha$ is no longer a free model parameter. In this case, Eq. (9) becomes $\rho_{\mathrm{u}}=\rho_{\mathrm{u} 0}$. Now, the solution for Eq. (18) is given by

$$
\rho_{\Lambda}=-3 \alpha \rho_{\mathrm{u} 0} \ln a+\text { const. },
$$

where const. is an integration constant. Then, inserting Eq. (22) into the Friedmann equation and requiring $E(z=0)=1$, one can determine the integration constant. Finally, we find that

$$
E^{2}=\Omega_{\mathrm{m} 0}(1+z)^{3}+3 \Omega_{\mathrm{u} 0}\left(1+\frac{1}{2 d_{\mathrm{u}}+1}\right) \ln (1+z)+\left(1-\Omega_{\mathrm{m} 0}\right) .
$$

In this case, there are 3 free model parameters, namely, $\Omega_{\mathrm{m} 0}, \Omega_{\mathrm{u} 0}$ and $d_{\mathrm{u}}$. By minimizing the corresponding total $\chi^{2}$ in Eq. (17), we find the best-fit parameters $\Omega_{\mathrm{m} 0}=0.270, \Omega_{\mathrm{u} 0}=0.020$ and $d_{\mathrm{u}}=0.273$, while $\chi_{\min }^{2}=313.168$. In Fig. 2. we present the corresponding $68 \%$ and $95 \%$ C.L. contours in the $\Omega_{\mathrm{u} 0}-d_{\mathrm{u}}$ plane for the interacting $\Lambda \mathrm{UCDM}$ model with $w_{\mathrm{u}}^{\text {eff }}=-1$, while the other parameters are taken to be the corresponding best-fit values. It is a pleasure to find that the unparticle dark component can have a considerable contribution to the total cosmic energy density $\left(\Omega_{\mathrm{u} 0}>0.1\right)$ in the $95 \%$ C.L. region while $1 \leq d_{\mathrm{u}} \leq 2$ (for bosonic unparticles) or $3 / 2 \leq d_{\mathrm{u}} \leq 5 / 2$ (for fermionic unparticles). The serious situation found in [11] could be relaxed in this case.

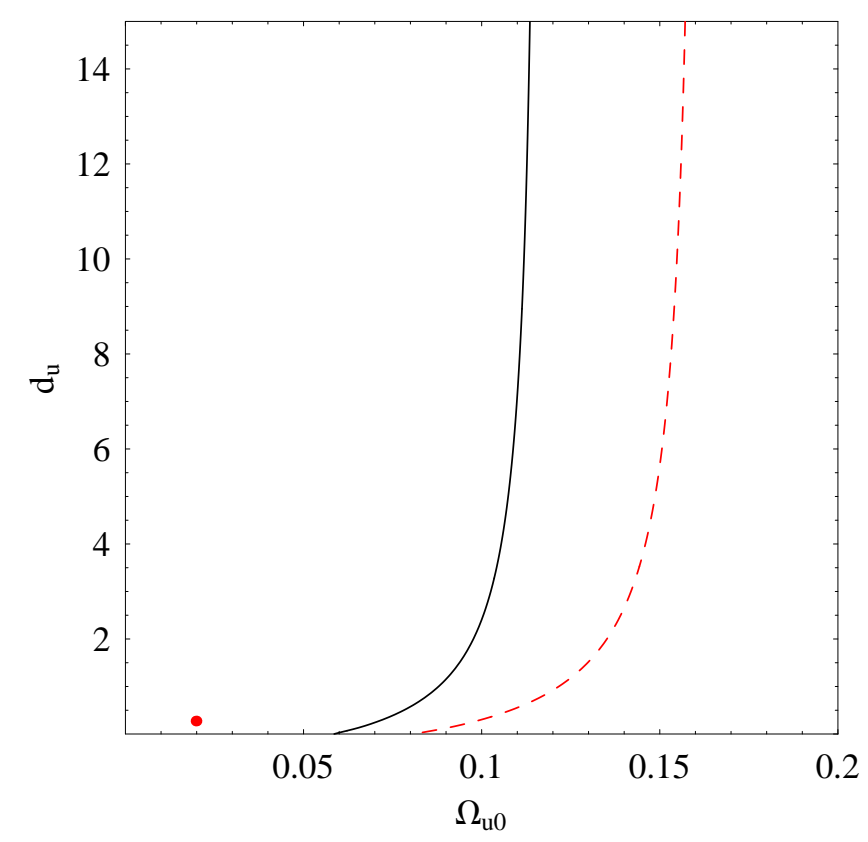

FIG. 2: The $68 \%$ C.L. (black solid line) and $95 \%$ C.L. (red dashed line) contours in the $\Omega_{\mathrm{u} 0}-d_{\mathrm{u}}$ plane for the interacting $\Lambda \mathrm{UCDM}$ model with $w_{\mathrm{u}}^{\mathrm{eff}}=-1$. The best fit is indicated by the red solid point. 


\section{COSMOLOGICAL CONSTRAINTS ON THE (INTERACTING) XUCDM MODEL}

In this section, we consider the (interacting) XUCDM model in which the EoS of dark energy $w_{\mathrm{x}}$ is a constant. We can recast Eq. (5) as

$$
\frac{d \rho_{\mathrm{x}}}{d a}+\frac{3}{a} \rho_{\mathrm{x}}\left(1+w_{\mathrm{x}}\right)=-\frac{3 \alpha}{a} \rho_{\mathrm{u} 0} a^{-3\left(1+w_{\mathrm{u}}^{\mathrm{eff}}\right)} .
$$

In the following, we first consider the case without interaction $(\alpha=0)$. Then, we consider two different cases with $w_{\mathrm{u}}^{\mathrm{eff}} \neq w_{\mathrm{x}}$ and $w_{\mathrm{u}}^{\mathrm{eff}}=w_{\mathrm{x}}$ for the differential equation (24).

\section{A. The XUCDM model without interaction}

It is natural to see first what will happen if there is no interaction between dark energy and unparticle dark component. In this case, $\alpha=0$. One can easily write down

$$
E^{2}=\Omega_{\mathrm{m} 0}(1+z)^{3}+\Omega_{\mathrm{u} 0}(1+z)^{3\left(1+w_{\mathrm{u}}\right)}+\left(1-\Omega_{\mathrm{m} 0}-\Omega_{\mathrm{u} 0}\right)(1+z)^{3\left(1+w_{\mathrm{x}}\right)} .
$$

There are 4 free model parameters, namely, $\Omega_{\mathrm{m} 0}, \Omega_{\mathrm{u} 0}, d_{\mathrm{u}}$ and $w_{\mathrm{x}}$. By minimizing the corresponding total $\chi^{2}$ in Eq. (17), we find the best-fit parameters $\Omega_{\mathrm{m} 0}=0.283, \Omega_{\mathrm{u} 0}=0.0, d_{\mathrm{u}}=0.540$ and $w_{\mathrm{x}}=-1.006$, while $\chi_{\text {min }}^{2}=311.981$. In Fig. 3, we present the corresponding $68 \%$ and $95 \%$ C.L. contours in the $\Omega_{\mathrm{u} 0}-d_{\mathrm{u}}$ plane and the $w_{\mathrm{x}}-d_{\mathrm{u}}$ plane for the XUCDM model without interaction, while the other parameters are taken to be the corresponding best-fit values. Obviously, from the left panel of Fig. 3, we see that the unparticle dark component can at most make up a few percent of the total cosmic energy density, so that it cannot be a major component in the case without interaction.
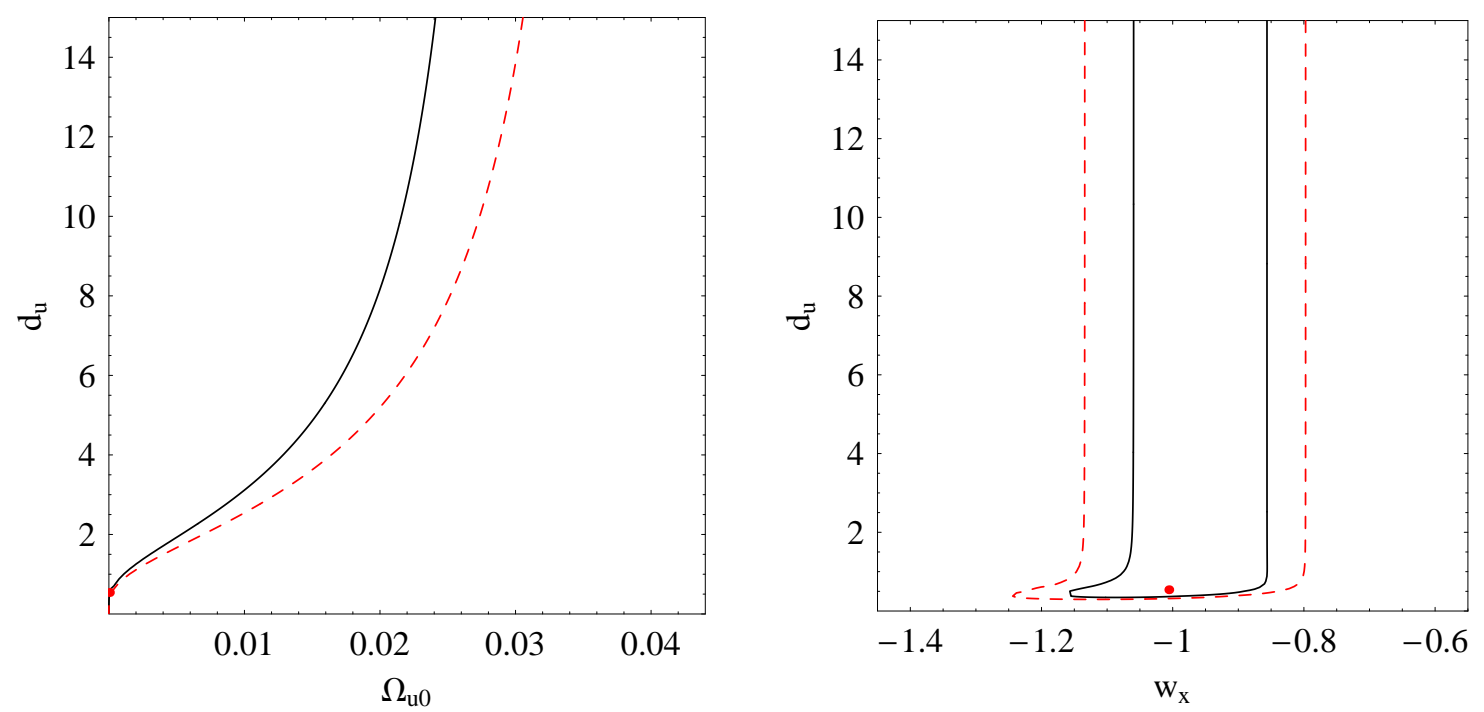

FIG. 3: The $68 \%$ C.L. (black solid lines) and $95 \%$ C.L. (red dashed lines) contours in the $\Omega_{\mathrm{u} 0}-d_{\mathrm{u}}$ plane and the $w_{\mathrm{x}}-d_{\mathrm{u}}$ plane for the XUCDM model without interaction. The best fit is indicated by the red solid point.

B. The interacting XUCDM model with $w_{\mathrm{u}}^{\text {eff }} \neq w_{\mathrm{x}}$

If $w_{\mathrm{u}}^{\mathrm{eff}} \neq w_{\mathrm{x}}$, the solution for Eq. (24) is given by

$$
\rho_{\mathrm{x}}=\frac{\alpha \rho_{\mathrm{u} 0}}{w_{\mathrm{u}}^{\text {eff }}-w_{\mathrm{x}}} a^{-3\left(1+w_{\mathrm{u}}^{\text {eff }}\right)}+\text { const. } \times a^{-3\left(1+w_{\mathrm{x}}\right)},
$$


where const. is an integration constant. Obviously, this solution diverges for $w_{\mathrm{u}}^{\text {eff }}=w_{\mathrm{x}}$ which will be considered in the next subsection. Inserting Eq. (26) into the Friedmann equation $H^{2}=\kappa^{2}\left(\rho_{\mathrm{x}}+\rho_{\mathrm{u}}+\rho_{\mathrm{m}}\right) / 3$ and requiring $E(z=0)=1$, one can determine the integration constant. Finally, we find that

$$
\begin{aligned}
E^{2}=\Omega_{\mathrm{m} 0}(1+z)^{3} & +\left(1+\frac{\alpha}{w_{\mathrm{u}}^{\text {eff }}-w_{\mathrm{x}}}\right) \Omega_{\mathrm{u} 0}(1+z)^{3\left(1+w_{\mathrm{u}}^{\text {eff }}\right)} \\
& +\left[1-\Omega_{\mathrm{m} 0}-\left(1+\frac{\alpha}{w_{\mathrm{u}}^{\text {eff }}-w_{\mathrm{x}}}\right) \Omega_{\mathrm{u} 0}\right](1+z)^{3\left(1+w_{\mathrm{x}}\right)} .
\end{aligned}
$$

There are 5 free model parameters, namely, $\Omega_{\mathrm{m} 0}, \Omega_{\mathrm{u} 0}, d_{\mathrm{u}}, \alpha$ and $w_{\mathrm{x}}$. By minimizing the corresponding total $\chi^{2}$ in Eq. (17), we find the best-fit parameters $\Omega_{\mathrm{m} 0}=0.277, \Omega_{\mathrm{u} 0}=0.214, d_{\mathrm{u}}=13.478, \alpha=0.589$, and $w_{\mathrm{x}}=-1.666$, while $\chi_{\min }^{2}=311.077$. In Fig. 4 we present the corresponding $68 \%$ and $95 \%$ C.L. contours in the $\Omega_{\mathrm{u} 0}-d_{\mathrm{u}}$ plane and the $\alpha-d_{\mathrm{u}}$ plane for the interacting XUCDM model with $w_{\mathrm{u}}^{\mathrm{eff}} \neq w_{\mathrm{x}}$, while the other parameters are taken to be the corresponding best-fit values. It is fortunate to find that the unparticle dark component can have a sizable contribution to the total cosmic energy density $\left(0.1<\Omega_{\mathrm{u} 0}<0.2\right.$ in the $68 \%$ C.L. region and $0.1<\Omega_{\mathrm{u} 0}<0.25$ in the $95 \%$ C.L. region $)$ while $1 \leq d_{\mathrm{u}} \leq 2$ (for bosonic unparticles) or $3 / 2 \leq d_{\mathrm{u}} \leq 5 / 2$ (for fermionic unparticles). The serious situation found in [11] could be significantly relaxed in this case.
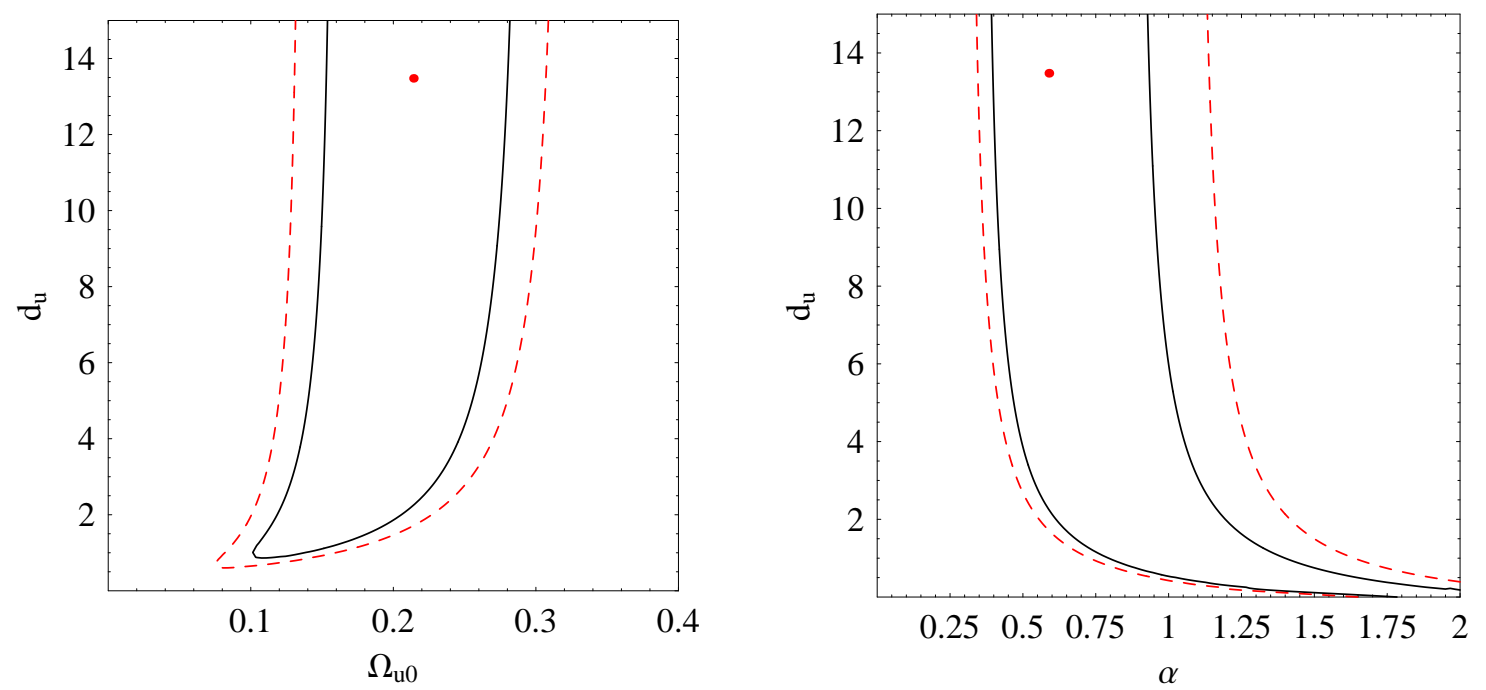

FIG. 4: The $68 \%$ C.L. (black solid lines) and 95\% C.L. (red dashed lines) contours in the $\Omega_{\mathrm{u} 0}-d_{\mathrm{u}}$ plane and the $\alpha-d_{\mathrm{u}}$ plane for the interacting XUCDM model with $w_{\mathrm{u}}^{\mathrm{eff}} \neq w_{\mathrm{x}}$. The best fit is indicated by the red solid point.

\section{The interacting XUCDM model with $w_{\mathrm{u}}^{\text {eff }}=w_{\mathrm{x}}$}

As mentioned above, the solution Eq. (26) diverges for $w_{\mathrm{u}}^{\mathrm{eff}}=w_{\mathrm{x}}$. Therefore, one is required to consider this case separately. If $w_{\mathrm{u}}^{\mathrm{eff}}=w_{\mathrm{x}}$, we find that

$$
\alpha=w_{\mathrm{u}}-w_{\mathrm{x}}=\frac{1}{2 d_{\mathrm{u}}+1}-w_{\mathrm{x}} .
$$

So, $\alpha$ is no longer a free model parameter. In this case, the solution for Eq. (24) is given by

$$
\rho_{\mathrm{x}}=\left(\text { const. }-3 \alpha \rho_{\mathrm{u} 0} \ln a\right) a^{-3\left(1+w_{\mathrm{x}}\right)},
$$


where const. is an integration constant. Inserting Eq. (29) into the Friedmann equation and requiring $E(z=0)=1$, one can determine the integration constant. Finally, we find that

$$
E^{2}=\Omega_{\mathrm{m} 0}(1+z)^{3}+\left[\left(1-\Omega_{\mathrm{m} 0}\right)+3 \Omega_{\mathrm{u} 0}\left(\frac{1}{2 d_{\mathrm{u}}+1}-w_{\mathrm{x}}\right) \ln (1+z)\right](1+z)^{3\left(1+w_{\mathrm{x}}\right)} .
$$

There are 4 free model parameters, namely, $\Omega_{\mathrm{m} 0}, \Omega_{\mathrm{u} 0}, d_{\mathrm{u}}$ and $w_{\mathrm{x}}$. By minimizing the corresponding total $\chi^{2}$ in Eq. (17), we find the best-fit parameters $\Omega_{\mathrm{m} 0}=0.270, \Omega_{\mathrm{u} 0}=0.0, d_{\mathrm{u}}=1.969$ and $w_{\mathrm{x}}=-0.954$, while $\chi_{\min }^{2}=313.138$. In Fig. 5 , we present the corresponding $68 \%$ and $95 \%$ C.L. contours in the $\Omega_{\mathrm{u} 0}-d_{\mathrm{u}}$ plane and the $w_{\mathrm{x}}-d_{\mathrm{u}}$ plane for the interacting XUCDM model with $w_{\mathrm{u}}^{\text {eff }}=w_{\mathrm{x}}$, while the other parameters are taken to be the corresponding best-fit values. It is a pleasure to find that the unparticle dark component can have a considerable contribution to the total cosmic energy density $\left(\Omega_{\mathrm{u} 0}>0.1\right)$ in the $95 \%$ C.L. region while $1 \leq d_{\mathrm{u}} \leq 2$ (for bosonic unparticles) or $3 / 2 \leq d_{\mathrm{u}} \leq 5 / 2$ (for fermionic unparticles). The serious situation found in [11] could also be relaxed in this case.
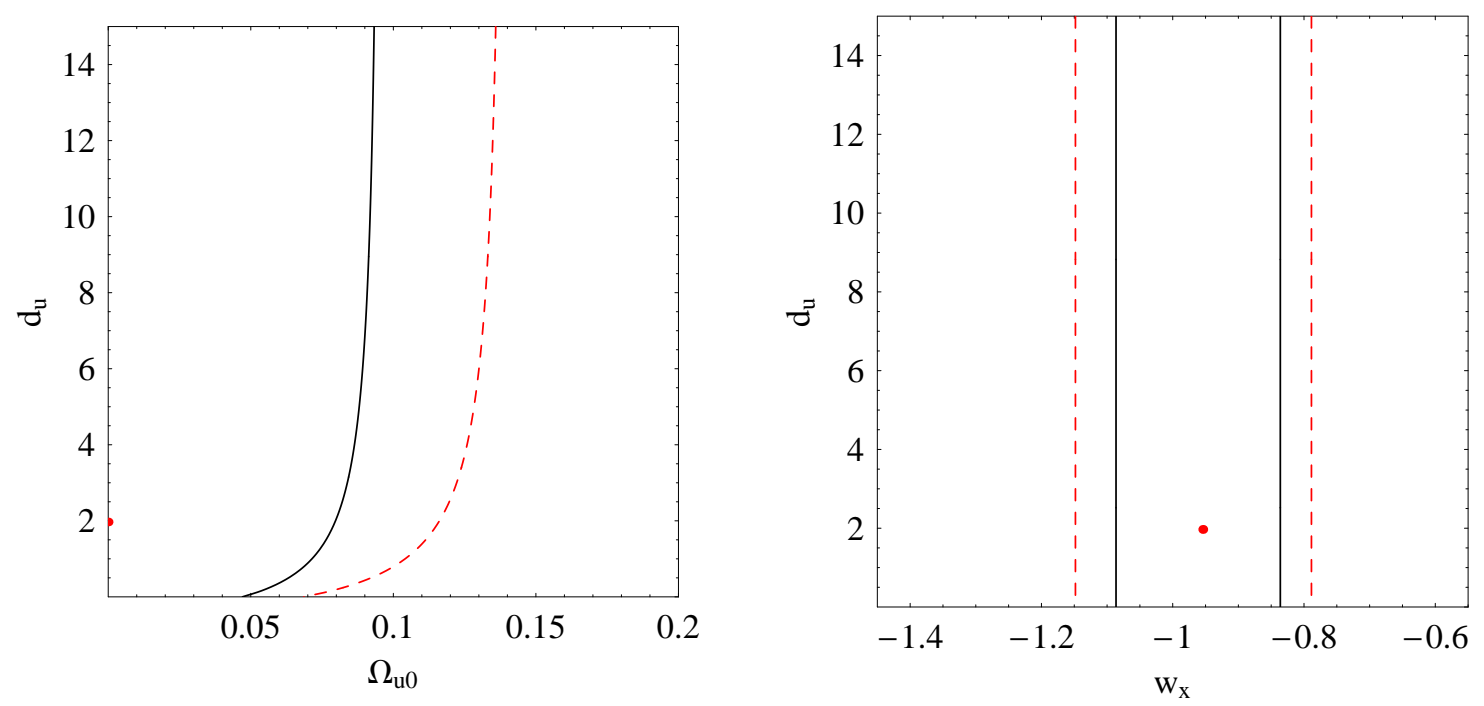

FIG. 5: The $68 \%$ C.L. (black solid lines) and 95\% C.L. (red dashed lines) contours in the $\Omega_{\mathrm{u} 0}-d_{\mathrm{u}}$ plane and the $w_{\mathrm{x}}-d_{\mathrm{u}}$ plane for the interacting XUCDM model with $w_{\mathrm{u}}^{\mathrm{eff}}=w_{\mathrm{x}}$. The best fit is indicated by the red solid point.

\section{CONCLUDING REMARKS}

Unparticle physics has been an active field since the seminal work of Georgi 1, 2]. Recently, many constraints on unparticles from various observations were considered in the literature. In particular, the cosmological constraints on the unparticle dark component put it in a serious situation [11]. In the present work, we try to find a way out of this serious situation, by including the possible interaction between dark energy and the unparticle dark component. In fact, as we have shown above, the cosmological constraints on the unparticle dark component can be relaxed in the interacting $\Lambda$ UCDM model with $w_{\mathrm{u}}^{\text {eff }}=-1$, and both the interacting XUCDM models with $w_{\mathrm{u}}^{\text {eff }} \neq w_{\mathrm{x}}$ and $w_{\mathrm{u}}^{\text {eff }}=w_{\mathrm{x}}$. In these interacting models, the unparticle dark component can have a considerable contribution to the total cosmic energy density (namely $\Omega_{\mathrm{u} 0}>0.1$; even $\Omega_{\mathrm{u} 0}$ can be larger than 0.2 in the interacting XUCDM model with $w_{\mathrm{u}}^{\text {eff }} \neq w_{\mathrm{x}}$ ) while $1 \leq d_{\mathrm{u}} \leq 2$ (for bosonic unparticles) or $3 / 2 \leq d_{\mathrm{u}} \leq 5 / 2$ (for fermionic unparticles), thanks to the possible interaction between dark energy and unparticle dark component. The tension between theoretical bounds and cosmological constraints could be removed. 
It is easy to understand these results physically. When dark energy, the major component of the universe, can decay into unparticles, the energy density of the unparticle dark component should decrease more slowly. Since both dark energy and unparticle are unseen, the interaction between them is not prevented. If the dilution rates of unparticle dark component and cold dark matter are comparable, it is possible to have a considerable energy density of the unparticle dark component at the present epoch, without requiring $d_{\mathrm{u}}$ to be very large. In fact, this is similar to the key point of the interacting dark energy models which are extensively considered in the literature to alleviate the cosmological coincidence problem (see e.g. [14, 25] and references therein).

As shown above, the cosmological constraints on the unparticle dark component can be relaxed in the interacting $\Lambda \mathrm{UCDM}$ model with $w_{\mathrm{u}}^{\mathrm{eff}}=-1$, and both the interacting XUCDM models with $w_{\mathrm{u}}^{\text {eff }} \neq w_{\mathrm{x}}$ and $w_{\mathrm{u}}^{\mathrm{eff}}=w_{\mathrm{x}}$. However, one might note that the unparticle dark component with cosmological constant is constrained by the observational data relatively more severe than in the case with dark energy. This is also easy to understand. Notice that the EoS of dark energy $w_{\mathrm{x}}$ is a free model parameter, whereas the EoS of the cosmological constant is a constant -1 . Therefore, the number of free model parameters in the interacting XUCDM model is larger than the one in the interacting $\Lambda$ UCDM model. As is well known, statistically, the constraints should be looser in this case.

\section{ACKNOWLEDGEMENTS}

We thank the anonymous referee for quite useful comments and suggestions, which helped us to improve this work. We are grateful to Professors Rong-Gen Cai, Shuang Nan Zhang, Xuelei Chen, and Xinmin Zhang, for helpful discussions. We also thank Minzi Feng, as well as Yan Gong, Xin Zhang and Dao-Jun Liu, for kind help and discussions. We would like to express our gratitude to IHEP (Beijing) for kind hospitality during the period of National Cosmology Workshop. This work was supported by the Excellent Young Scholars Research Fund of Beijing Institute of Technology.

[1] H. Georgi, Phys. Rev. Lett. 98, 221601 (2007) hep-ph/0703260.

[2] H. Georgi, Phys. Lett. B 650, 275 (2007) arXiv:0704.2457.

[3] K. Cheung, W. Y. Keung and T. C. Yuan, AIP Conf. Proc. 1078, 156 (2009) arXiv:0809.0995.

[4] A. Rajaraman, AIP Conf. Proc. 1078, 63 (2009) arXiv:0809.5092.

[5] K. Cheung, W. Y. Keung and T. C. Yuan, Phys. Rev. Lett. 99, 051803 (2007) arXiv:0704.2588;

K. Cheung, W. Y. Keung and T. C. Yuan, Phys. Rev. D 76, 055003 (2007) arXiv:0706.3155;

K. Cheung, W. Y. Keung and T. C. Yuan, arXiv:0710.2230 [hep-ph].

[6] Y. Liao and J. Y. Liu, Phys. Rev. Lett. 99, 191804 (2007) arXiv:0706.1284;

H. Goldberg and P. Nath, Phys. Rev. Lett. 100, 031803 (2008);

N. G. Deshpande, S. D. H. Hsu and J. Jiang, Phys. Lett. B 659, 888 (2008) arXiv:0708.2735.

[7] A. Freitas and D. Wyler, JHEP 0712, 033 (2007) arXiv:0708.4339.

[8] M. C. Gonzalez-Garcia et al., JCAP 0806, 019 (2008) arXiv:0803.1180;

L. Anchordoqui and H. Goldberg, Phys. Lett. B 659, 345 (2008) arXiv:0709.0678.

[9] H. Davoudiasl, Phys. Rev. Lett. 99, 141301 (2007) arXiv:0705.3636;

S. Hannestad, G. Raffelt and Y. Y. Y. Wong, Phys. Rev. D 76, 121701 (2007) arXiv:0708.1404;

P. K. Das, Phys. Rev. D 76, 123012 (2007) arXiv:0708.2812;

S. Dutta and A. Goyal, JCAP 0803, 027 (2008) arXiv:0712.0145.

[10] J. McDonald, arXiv:0709.2350 [hep-ph];

J. McDonald, arXiv:0805.1888 [hep-ph];

I. Lewis, arXiv:0710.4147 [hep-ph];

B. Grzadkowski and J. Wudka, arXiv:0809.0977 [hep-ph].

[11] Y. Gong and X. Chen, Eur. Phys. J. C 57, 785 (2008) arXiv:0803.3223. 
[12] S. L. Chen, X. G. He, X. P. Hu and Y. Liao, Eur. Phys. J. C 60, 317 (2009) arXiv:0710.5129.

[13] N. G. Deshpande, X. G. He and J. Jiang, Phys. Lett. B 656, 91 (2007) arXiv:0707.2959;

T. Kikuchi and N. Okada, Phys. Lett. B 665, 186 (2008) arXiv:0711.1506.

[14] H. Wei and R. G. Cai, Phys. Rev. D 71, 043504 (2005) hep-th/0412045;

H. Wei and R. G. Cai, Phys. Rev. D 72, 123507 (2005) astro-ph/0509328;

H. Wei and R. G. Cai, Phys. Rev. D 73, 083002 (2006) astro-ph/0603052;

H. Wei and R. G. Cai, JCAP 0709, 015 (2007) astro-ph/0607064;

H. Wei and S. N. Zhang, Phys. Lett. B 644, 7 (2007) astro-ph/0609597];

H. Wei and S. N. Zhang, Phys. Lett. B 654, 139 (2007) arXiv:0704.3330;

H. Wei and R. G. Cai, Phys. Lett. B 655, 1 (2007) arXiv:0707.4526;

H. Wei and S. N. Zhang, Phys. Rev. D 78, 023011 (2008) arXiv:0803.3292;

H. Wei and R. G. Cai, Eur. Phys. J. C 59, 99 (2009) arXiv:0707.4052.

[15] M. Kowalski et al., Astrophys. J. 686, 749 (2008) arXiv:0804.4142.

The numerical data of the full sample are available at http://supernova.lbl.gov/Union

[16] S. Nesseris and L. Perivolaropoulos, Phys. Rev. D 72, 123519 (2005) astro-ph/0511040;

L. Perivolaropoulos, Phys. Rev. D 71, 063503 (2005) astro-ph/0412308.

[17] E. Di Pietro and J. F. Claeskens, Mon. Not. Roy. Astron. Soc. 341, 1299 (2003) astro-ph/0207332.

[18] J. R. Bond, G. Efstathiou and M. Tegmark, Mon. Not. Roy. Astron. Soc. 291, L33 (1997) astro-ph/9702100.

[19] Y. Wang and P. Mukherjee, Astrophys. J. 650, 1 (2006) astro-ph/0604051.

[20] E. Komatsu et al. [WMAP Collaboration], Astrophys. J. Suppl. 180, 330 (2009) arXiv:0803.0547.

[21] M. Tegmark et al. [SDSS Collaboration], Phys. Rev. D 69, 103501 (2004) astro-ph/0310723;

M. Tegmark et al. [SDSS Collaboration], Astrophys. J. 606, 702 (2004) astro-ph/0310725;

U. Seljak et al. [SDSS Collaboration], Phys. Rev. D 71, 103515 (2005) astro-ph/0407372;

M. Tegmark et al. [SDSS Collaboration], Phys. Rev. D 74, 123507 (2006) astro-ph/0608632.

[22] D. J. Eisenstein et al. [SDSS Collaboration], Astrophys. J. 633, 560 (2005) astro-ph/0501171].

[23] D. C. Dai and D. Stojkovic, arXiv:0812.3396 [gr-qc].

[24] F. Sannino and R. Zwicky, Phys. Rev. D 79, 015016 (2009) arXiv:0810.2686.

[25] Z. K. Guo, N. Ohta and S. Tsujikawa, Phys. Rev. D 76, 023508 (2007) astro-ph/0702015.

[26] J. M. Overduin and F. I. Cooperstock, Phys. Rev. D 58, 043506 (1998) astro-ph/9805260;

J. M. Overduin and P. S. Wesson, Phys. Rept. 402, 267 (2004) astro-ph/0407207.

[27] P. Wang and X. H. Meng, Class. Quant. Grav. 22, 283 (2005) astro-ph/0408495.

[28] Y. Z. Ma, Nucl. Phys. B 804, 262 (2008) arXiv:0708.3606. 